\title{
REGION AND NUMBER OF PERIODIC SOLUTIONS OF DUFFING'S EQUATION
}

\author{
By YASUNORI SAWANO
}

\section{$\S 1$. Introduction.}

In this paper, we consider the generalized equation

$$
x^{\prime \prime}+f(x) x^{\prime}+g(x)=e(t), \quad,=\frac{d}{d t}
$$

of Duffing's equation

$$
x^{\prime \prime}+c x^{\prime}+a x+b x^{3}=\varepsilon \cos \omega t .
$$

where $a, b, c$ and $\varepsilon$ are positive constants. Suppose that $f(x)$ and $g(x)$ in the equation (1.1) satisfy the following conditions:

$$
f(x), g(x) \in C^{2}(R) \text {; }
$$

$$
e(t) \text { is periodic with the least period } L \text {; }
$$

$$
|e(t)| \leqq E \quad \text { for }{ }^{\forall} t \text {, }
$$

where $E$ is a positive constant.

In section 2, we indicate in Theorem 1 the existence of periodic solutions of the equation (1.1) and the regions where the periodic solutions exist. The analogous results have been obtained by N. Levinson [1], [2], W. S. Loud [4] and K. Shiraiwa [5], when $d g(x) / d x$ is positive for any $x$, and $x g(x)$ is not negative for any $x$. Moreover, Levinson [1] and [2] assumed that $f(x)$ is positive for $|x| \geqq X$, where $X$ is a positive constant. Loud [4] and Shiraiwa [5] supposed that $f(x)$ is positive. We show the statements under weaker assumptions. In our proof, we applied the method of Shiraiwa [5].

In section 3 , we show in Theorem 8 that the periodic solutions which are given in Theorem 1 are completely stable when we assume $f(x)>d g(x) / d x>1$ in some region. This property was showed by Levinson [1] when $g(x)=x$, and by Loud [4] and Shiraiwa [5] when $f(x)$ is a positive constant. We can show it by the same way as in Levinson [1] except how to take the distance of two different solutions of the equation (1.1).

Received May 7, 1986 
In section 4, we give, as the application of the previous sections, the number of periodic solutions, their property and the regions where they exist, and in the last section, numerical results are given where the statement of section 4 are asserted for two particular equations.

\section{§2. Existence of periodic solutions.}

Up to the present, many people have shown the existence of periodic solutions of the equation (1.1) with the following assumptions for $f(x), g(x)$ :

$$
\begin{array}{ll}
f(x) \geqq C>0 & \text { for }|x| \geqq{ }^{\exists} A>0 \\
\frac{d g}{d x}(x)>0 & \text { for }{ }^{\forall} x \in R \\
x g(x)>0 & \text { for } x \neq 0 .
\end{array}
$$

Beside K. Shiraiwa [5] indicated a region where the periodic solution exists under the assumptions :

$$
\begin{aligned}
& f(x)>C>0 \quad \text { for }{ }^{\forall} x \in R ; \\
& 0 \leqq \frac{d g}{d x}(x)<{ }^{\exists} M \quad \text { for }|x|{ }^{\forall} m ; \\
& \lim _{x \rightarrow \infty}|g(x)|>E,
\end{aligned}
$$

where $M$ depends on $m$. In this paper, we prove the existence of periodic solutions of the equation (1.1) under weaker assumption, and we prove the regions where these solutions are.

THEOREM 1. Assume the condition (1.3). We suppose that, there exist $\alpha$ and $\beta$ such that

$$
\begin{aligned}
& g(\alpha)=-E \\
& g(\beta)=E \\
& |g(x)| \leqq E \quad \text { for } \alpha<x<\beta .
\end{aligned}
$$

Moreover, assume that $f(x)$ and $g(x)$ satısfy

$$
\begin{array}{ll}
f(x) \geqq C>0 & \text { for } \alpha-\frac{4 E}{C^{2}} \leqq x \leqq \beta+\frac{4 E}{C^{2}} \\
\frac{d g}{d x}(x) \geqq 0 & \text { for } \alpha-\frac{4 E}{C^{2}} \leqq x \leqq \alpha, \beta \leqq x \leqq \beta+\frac{4 E}{C^{2}} .
\end{array}
$$

Then the equation (1.1) has at least one periodic solution with the least period $L$ in the region 


$$
D=\left\{\left(x, x^{\prime}\right)\left|\alpha-\frac{4 E}{C^{2}}<x<\beta+\frac{4 E}{C^{2}},\right| x^{\prime} \mid \leqq \frac{4 E}{C}\right\} .
$$

Proof. We consider the system

$$
\begin{aligned}
& x^{\prime}=y \\
& y^{\prime}=-f(x) y-g(x)+e(t),
\end{aligned}
$$

which is equivalent to the equation (1.1). From the equation (2.3), we have

$$
y \frac{d y}{d x}=-f(x) y-g(x)+e(t) .
$$

Here we define $d y / d x$ at $y=0$ as

$$
\frac{d y}{d x}=\left\{\begin{aligned}
+\infty & \text { for } y^{\prime}>0 \\
0 & \text { for } y^{\prime}=0 \\
-\infty & \text { for } y^{\prime}<0 .
\end{aligned}\right.
$$

We define the following equations:

$$
\begin{array}{ll}
y \frac{d y}{d x}=-C y-g(x)+E & y \geqq 0 \\
y \frac{d y}{d x}=-C y-g(x)-E & y \leqq 0,
\end{array}
$$

where we assume also that $d y / d x$ satisfy (2.5). From the first inequalities in the assumption (2.2), we have

$$
\begin{array}{ll}
\frac{d y}{d x} \text { in }(2.4) \leqq \frac{d y}{d x} \text { in (2.6) } & y>0 \\
\frac{d y}{d x} \text { in }(2,4) \leqq \frac{d y}{d x} \text { in }(2.7) & y<0 .
\end{array}
$$

We need the following lemmas for solutions of the equation (2.6), whose proofs will be given after the proof of Theorem1.

LEMMA 2. All the solutions $y(x)$ of the equation (2.6) have the following properties in the upper half plane $\{(x, y) \mid y>0\}$ :

(2.10.1) $\quad y(x)$ is a increasing function of $x$ below the curve $-C y-g(x)+E=0$, and decreasing above it.;

(2.11.1) $y(x)$ have extremums at the point which is the meeting point of the curve $-C y-g(x)+E=0$.; 
(2.12.1) $y(x)$ which start the region $\left\{(x, y) \mid \alpha-4 E / C^{2}<x<\beta, y>0\right\}$, meet with the line $x=\beta$ in the upper half plane.

LEMMA 3. All the solutions $y(x)$ of the equation (2.7) have the following properties in the lower half plane $\{(x, y) \mid y<0\}$ :

(2.10.2) $y(x)$ is a decreasing function of $x$ above the curve $-C y-g(x)-E=0$, and increasing below it. ;

(2.11.2) $\quad y(x)$ have extremums at the point which is the meeting point of the curve $-C y-g(x)-E=0$. ;

(2.12.2) $y(x)$ which start the region $\left\{(x, y) \mid \alpha<x<\beta+4 E / C^{2}, y<0\right\}$, meet with the line $x=\alpha$ in the lower half plane.

Moreover, we assume the next lemmas.

LEMMA 4. The solutions of the equation (2.6) starting from $(\beta, 4 E / C)$ intersect with $x$-axis at some $x$ such that $\beta<x<\beta+4 E / C^{2}$.

LEMMA 5. The solutions of the equation (2.7) starting from $(\alpha,-4 E / C)$ intersect with $x$-axis at some $x$ such that $\alpha-4 E / C^{2}<x<\alpha$.

From now on, we consider the domain bounded by the following curves $C_{1}-C_{10}$.

$C_{1}$ is the solution of the equation (2.6) started from $(\beta, 4 E / C)$ to a intersection with $x$-axis at $\left(x_{1}, 0\right)$. Lemma 4 gives that $\beta<x_{1}<\beta+4 E / C^{2}$. Next we define $C_{2}$ as the solution of the equation (2.7) started from $\left(x_{1}, 0\right)$ to a intersection with the curve $-C y-g(x)-E=0$. Let the intersection be called $\left(x_{2}, y_{2}\right)$. Here, we suppose the next lemma, too.

LEMMA 6. The point $\left(x_{2}, y_{2}\right)$ satisfies $y_{2} \geqq-4 E / C$.

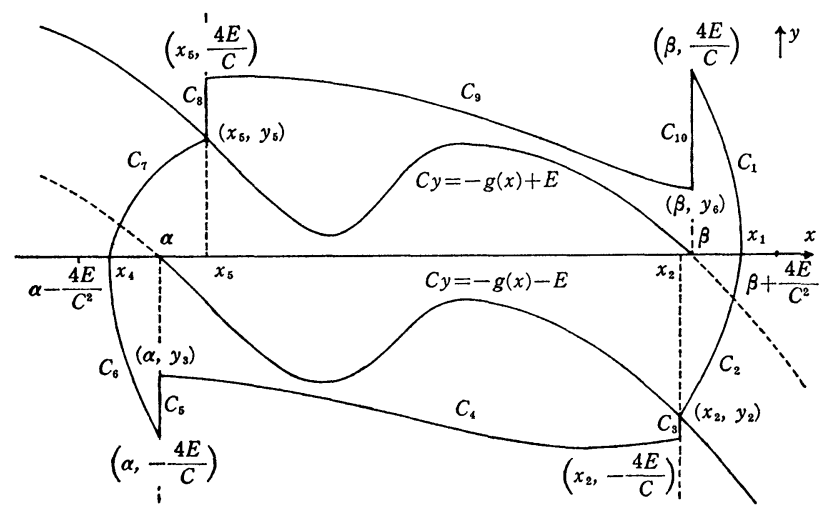

Fig. 1. 
From this lemma we have $y_{2} \geqq-4 E / C$, then we define $C_{3}$ as the line segment from $\left(x_{2}, y_{2}\right)$ to $\left(x_{2},-4 E / C\right)$. Next we make the curve $C_{4}$, which is the solution of the equation (2.7) starting from $\left(x_{2},-4 E / C\right)$ till $x=\alpha$. The end point is $\left(\alpha, y_{3}\right)$. It is clear that $\left(x_{2},-4 E / C\right)$ lies below the curve $-C y-g(x)-E$ $=0$. So the solution of the equation (2.7) is increasing in some interval. The assumption (2.2) gives $d g(x) / d x>0$ at $\beta<x<\beta+4 E / C^{2}$, then the curve $-C y-g(x)-E=0$ is decreasing in $\beta<x<\beta+4 E / C^{2}$. From this and the assumption, we have

$$
0>y>-2 E / C \quad \text { for } \alpha<x<\beta,
$$

where $(x, y)$ is the point on the curve $-C y-g(x)-E=0$. Then we have $y \geqq$ $-4 E / C$ by Lemma 3. At the end, we define $C_{5}$, the line segment from $\left(\alpha, y_{3}\right)$ to $(\alpha,-4 E / C)$. And, we assume the following Lemma 7 for drawing the curves $C_{6}-C_{10}$ by the same way.

Lemma 7. The point $\left(x_{5}, y_{5}\right)$ satısfies $y_{5} \leqq 4 E / C$.

We consider the tangent vectors of the solutions of the equation (2.4) on these curves $C_{1}, C_{2}, C_{4}, C_{6}, C_{7}$ and $C_{9}$. From the equations (2.8) and (2.9), we find that they return to the inside of the region $D$. For $C_{3}, C_{5}, C_{8}$ and $C_{10}$, the first equation of (2.3) shows that the tangent vectors direct inside of it. Hence, any solution of the equation (2.4) enter the domain $D$, and never go out from $D$. Therefore, the equation (2.6) has at least one periodic solution with the least period $L$ by Brower's fixed point theorem. Now, we completed the proof of Theorem 1.

From now, we prove the lemmas.

Proof of Lemma 2. From the equation (2.6), we get $d y / d x<0, d y / d x>0$ and $d y / d x=0$ respectively below, above and on the curve $-C y-g(x)+E=0$. From these, we have (2.10.1) and (2.11.1).

(2.11.1) shows that the solution of the equation (2.6) started from $\{(x, y) \mid \alpha$ $\left.-4 E / C^{2}<x<\beta, y>0\right\}$ has its infimum in this region $D$ at one of the following point which is the meeting point with the curve $-C y-g(x)+E=0$ or $x=\beta$ or starting point. But in this domain the curve $-C y-g(x)+E=0$ is above the $x$-axis. Hence, the solutions of the equation (2.6) under consideration meet $x=\beta$ in the upper half plane. The proof is completed.

Proof of Lemma 4. The assumptions (2.1) and (2.2) give

$$
g(x) \geqq E \quad \text { for } \beta \leqq x \leqq \beta+\frac{4 E}{C^{2}},
$$

and from

$$
y \frac{d y}{d x}=-C y-g(x)+E,
$$


we have $d y / d x<0$. Now, we multiply the equation (2.6) by $y$, next we differentiate the product with respect to $x$, then we find

$$
y^{2} \frac{d^{2} y}{d x^{2}}=(g(x)-E) \frac{d y}{d x}-\frac{d g}{d x}(x) y .
$$

Here $g(x) \geqq E, d y / d x \leqq 0, d g(x) / d x \leqq 0$ and $y \geqq 0$, so we find $d^{2} y / d x^{2} \leqq 0$. This gives the solutions of the equation (2.6) that are concave downward.

These show that the solutions of the equation (2.6) are below the tangent line of the solution through $(\beta, 4 E / C)$ in $\beta<x<\beta+4 E / C^{2}$. Hence, we have proved Lemma 4.

Proof of Lemma 6. When $x_{2} \leqq \beta$, it is clear that $y_{2}>-4 E / C$ from $g(x)<E$, because $\left(x_{2}, y_{2}\right)$ is the cross point of $-C y-g(x)-E=0$ and the solution of the equation (2.7). So we must show the lemma for $x_{2}>\beta$. From Lemma 2, we know that $y_{2}$ is the minimum of $y$ of $C_{2}$.

Now we integrate the equation (2.6) from $(\beta, 4 E / C)$ to $\left(x_{1}, 0\right)$. So we have

$$
-\frac{r^{2}}{2}=-C \int_{\beta}^{x_{1}} y d x-G\left(x_{1}\right)+G(\beta)+E\left(x_{1}-\beta\right) .
$$

Here $r=4 E / C$ and

$$
G(x)=\int_{0}^{x} g(u) d u .
$$

$C_{1}$ is the solution of the equation (2.13), so it is convex upward. Then the integral part of the equation (2.14) is greater than the area of triangle whose vertices are $(\beta, 4 E / C),(\beta, 0)$ and $\left(x_{1}, 0\right)$. Hence, we find

$$
-\frac{r^{2}}{2} \leqq-C \frac{r}{2}\left(x_{1}-\beta\right)-G\left(x_{1}\right)+G(\beta)+E\left(x_{1}-\beta\right) .
$$

On the other hand, we integrate the equation (2.7) from $\left(x_{1}, 0\right)$ to $\left(x_{2}, y_{2}\right)$. Then we have

$$
\frac{y_{2}^{2}}{2}=-C \int_{x_{1}}^{x_{2}} y d x-G\left(x_{2}\right)+G\left(x_{1}\right)-E\left(x_{2}-x_{1}\right) .
$$

The integrated part of the equation (2.16) is positive when $y<0$ and $x_{2}<x_{1}$. Therefore the equation (2.16) gives

$$
\frac{y_{2}^{2}}{2} \leqq-G\left(x_{2}\right)+G\left(x_{1}\right)-E\left(x_{2}-x_{1}\right) \text {. }
$$

We add the equation (2.15) to the equation (2.17). So we find

$$
\begin{aligned}
\frac{\left(y_{2}^{2}-r^{2}\right)}{2} & \leqq-C \frac{r}{2}\left(x_{1}-\beta\right)-G\left(x_{2}\right)+G(\beta)+E\left[\left(x_{1}-\beta\right)+\left(x_{1}-x_{2}\right)\right] \\
& \leqq\left(-C \frac{r}{2}+2 E\right)\left(x_{1}-\beta\right)-\int_{\beta}^{x_{2}} g(u) d u \leqq 0
\end{aligned}
$$


Here, from $r=4 E / C,\left(x_{1}-\beta\right)>\left(x_{1}-x_{2}\right)$ and the conditions (2.1) and (2.2), we have

$$
\int_{\beta}^{x_{2}} g(u) d u \leqq 0
$$

Hence, we find

$$
y_{2}^{2}-r^{2} \leqq 0
$$

After all, we have $\left|y_{2}\right| \leqq r=4 E / C$, so the proof of Lemma 6 is completed. Lemma 3, Lemma 5 and Lemma 7 are proved in the same way as the proof of Lemma 2, Lemma 4 and Lemma 6 respectively.

\section{$\S 3$. Uniqueness of periodic solutions in some region}

In the previous section, we show the existence of periodic solutions. In this section, we prove that there is only one periodic solution which is completely stable in some region.

In this section, we consider the following system:

$$
\begin{aligned}
& x^{\prime}=y-F(x) \\
& y^{\prime}=-g(x)+e(t)
\end{aligned}
$$

which is equivalent to the equation (1.1). Here, we define

$$
F(x)=\int_{0}^{x} f(u) d u .
$$

Let $(X(t), Y(t))$ be the periodic solution of the equation (3.1) with the least period $L$. We consider the following transformation $T$ :

$$
T:\left(x\left(t_{0}\right), y\left(t_{0}\right)\right) \longrightarrow\left(x\left(t_{0}+L\right), y\left(t_{0}+L\right)\right) .
$$

Let $\lambda_{1}, \lambda_{2}$ be the eigenvalue of the Jacobian matrix of the transformation $T$ at $\left(X\left(t_{0}\right), Y\left(t_{0}\right)\right)$. The periodic solution, $(X(t), Y(t))$ (or the fixed point, $\left.\left(X\left(t_{0}\right), Y\left(t_{0}\right)\right)\right)$ is called completely stable if $\left|\lambda_{1}\right|<1,\left|\lambda_{2}\right|<1$.

Similarly if $\lambda_{1}>1>\lambda_{2}>0$, then we shall call the solution $(X(t), Y(t))$, directly unstable.

THEOREM 8. In addition to the assumptions of Theorem 1, assume that $f(x)$ and $g(x)$ satisfy

$$
f(x) \geqq \frac{d g}{d x}(x)>1 \quad \text { for } \forall x \in D .
$$

Then, in the region $D$ given in Theorem 1 , there exists only one periodic solution of the equation (1.1) with the least preriod $L$ and it is completely stable.

Proof. We assume the following lemma. 
LEMMA 9. Let $\left(x_{1}(t), y_{1}(t)\right)$ and $\left(x_{2}(t), y_{2}(t)\right)$ be two different solutions which are contained in $D$. We define a function $\rho(t)$ by

$$
\rho(t)= \begin{cases}\sqrt{\left(\left(x_{2}(t)-x_{1}(t)\right)^{2}+\left(y_{2}(t)-y_{1}(t)\right)^{2}\right.} & \text { for } B \geqq 0 \\ \left|x_{2}(t)-x_{1}(t)\right|+\left|y_{2}(t)-y_{1}(t)\right| & \text { for } B \leqq 0 .\end{cases}
$$

where

$$
B=\left(x_{2}(t)-x_{1}(t)\right)\left(y_{2}(t)-y_{1}(t)\right) .
$$

Then $\rho(t)$ is a decreasing function in $(0, \infty)$.

The proof of this lemma will be sent after the proof of Theorem 8 .

Let $X(t)$ be a periodic solution of the equation (1.1). By substituting $X(t)$ into the equation (1.1) it follows at once the period of $X(t)$, which is denoted by $L_{1}$, must be some multiple of $L$. Here, we assume that there exists a solution $x_{0}(t)$ of the equation (1.1) which does not tend to $X(t)$ as $t \rightarrow \infty$ in the region $D$.

Corresponding to $X(t)$ and $x_{0}(t)$ we have solutions $(X(t), Y(t))$ and $\left(x_{0}(t), y_{0}(t)\right)$ of the equation (3.1) respectively. Since $x_{0}(t)$ does not tend to $X(t)$, we have that $\left(x_{0}(t), y_{0}(t)\right)$ does not tend to $(X(t), Y(t))$ as $t \rightarrow \infty$. Then we denote the distance of $(X(t), Y(t))$ and $\left(x_{0}(t), y_{0}(t)\right)$ by $\rho_{0}(t)$.

We have

$$
\lim _{t \rightarrow \infty} \rho_{0}(t)=A>0 .
$$

We denote the distance between $\left(X\left(t+n L_{1}\right), Y\left(t+n L_{1}\right)\right)$, and $\left(x_{0}\left(t+n L_{1}\right), y_{0}\left(t+n L_{1}\right)\right)$ by $\rho_{n}(t)$. Then, we find

Then we have

$$
\rho_{n}(t)=\rho_{0}\left(t+n L_{1}\right)
$$

$$
\lim _{n \rightarrow \infty} \rho_{n}(t)=A
$$

from (3.6). When we denote the points $(X(0), Y(0))$ and $\left(x_{0}\left(n L_{1}\right), y_{0}\left(n L_{1}\right)\right)$ by $P$ and $P_{n}$, respectively, the distance $P_{n} P$ must approach to $A$ as $t \rightarrow \infty$ from (3.7).

From this fact, there exists a positive integer $N$ such that

$$
P_{n} P<A+\varepsilon \quad \text { for } n \geqq N,
$$

for any $\varepsilon$. Then we take any $n_{1}$ and such that $n_{1}>N$ and any $\delta$ such that $A>\delta>0$ and $Q_{n_{1}}$ denote the point whose distance from $P$ is $\delta$, and it is on the line $P_{n_{1}} P$. That is

So we have

$$
Q_{n_{1}} P=\delta
$$

$$
Q_{n_{1}} P_{n_{1}}=A+\varepsilon-\delta .
$$

Now we denote the solution which starts $Q_{n_{1}}$ by $\left(x_{1}(t), y_{1}(t)\right)$, then from Lemma 9 we find 


$$
\begin{aligned}
& \lim _{n \rightarrow \infty} P_{n} P=A \\
& \lim _{n \rightarrow \infty} Q_{n} P=A_{1}<\delta \\
& \lim _{n \rightarrow \infty} Q_{n} P_{n}<A+\varepsilon-\delta .
\end{aligned}
$$

And we know

$$
P_{n} P<P_{n} Q_{n}+Q_{n} P,
$$

then we have

$$
A<A_{1}+(A+\varepsilon-\delta)
$$

by the equation (3.8). Here, $\varepsilon$ is arbitrary, so we find

$$
A \leqq A+\left(A_{1}-\delta\right) \text {. }
$$

This is a contradiction with $A_{1}<\delta$. Hence, (3.6) and (3.7) do not hold. This proves that the periodic solution $X(t)$ of the equation (1.1) in the domain $D$ is completely stable.

Next we show that $L$ is the period of this solution. We suppose $L_{1} \neq L$. Then $(X(t), Y(t))$ and $(X(t-L), Y(t-L))$ is a periodic solutions of the equation (3.1). But $(X(t-L), Y(t-L))$ does not tend to $(X(t), Y(t))$ as $t \rightarrow \infty$. Therefore, this contradict to Lemma 9. Then we can say that the least period of $(X)(t), Y(t))$ is $L$. Now, the proof of Theorem 8 is completed.

Proof of Lemma 9. We consider the solution $(x(t), y(t))$ in a neighborhood of $t \in(0, \infty)$ such that $\left(x_{2}(t)-x_{1}(t)\right)>0$ and $\left(y_{2}(t)-y_{1}(t)\right)>0$. We differentiate $\rho(t)$ with respect to $t$, so we find

$$
\rho(t) \rho^{\prime}(t)=\left(x_{2}(t)-x_{1}(t)\right)\left(x_{2}^{\prime}(t)-x_{1}^{\prime}(t)\right)+\left(y_{2}(t)-y_{1}(t)\right)\left(y_{2}^{\prime}(t)-y_{1}^{\prime}(t)\right) .
$$

Here we have

$$
\begin{gathered}
\left(x_{2}(t)-x_{1}(t)\right)\left(x_{2}^{\prime}(t)-x_{1}^{\prime}(t)\right)+\left(y_{2}(t)-y_{1}(t)\right)\left(y_{2}^{\prime}(t)-y_{1}^{\prime}(t)\right) \\
=\left(y_{2}(t)-y_{1}(t)\right) \int_{x_{1}(t)}^{x_{2}(t)}\left(1-\frac{d g}{d x}(x)\right) d x \\
-\left(x_{2}(t)-x_{1}(t)\right) \int_{x_{1}(t)}^{x_{2}(t)} f(x) d x
\end{gathered}
$$

which is negative. From the assumption $1<d g(x) / d x$. Hence, $\rho(t)>0$ gives

$$
\rho^{\prime}(t)<0
$$

by the equation (3.11).

We can find the proof as the same way when we assume $\left(x_{2}(t)-x_{1}(t)\right)<0$ and $\left(y_{2}(t)-y_{1}(t)\right)<0$.

Next we consider the solution $(x(t), y(t))$ in a neighborhood of $t \in(0, \infty)$ 
such that $\left(x_{2}(t)-x_{1}(t)\right)>0$ and $\left(y_{2}(t)-y_{1}(t)\right)<0$. We find

$$
\rho(t)=\left(x_{2}(t)-x_{1}(t)\right)-\left(y_{2}(t)-y_{1}(t)\right) .
$$

We differentiate this equation with respect to $t$, and get

$$
\begin{aligned}
\rho^{\prime}(t) & =\left(x_{2}^{\prime}(t)-x_{1}^{\prime}(t)\right)-\left(y_{2}^{\prime}(t)-y_{1}^{\prime}(t)\right) \\
& =\left(y_{2}(t)-y_{1}(t)\right)-\int_{x_{1}(t)}^{x_{2}(t)}\left(f(x)-\frac{d g}{d x}(x)\right) d x .
\end{aligned}
$$

The assumption $(f(x)-d g(x) / d x) \geqq 0$ gives

$$
\rho^{\prime}(t)<0 \text {. }
$$

And, we can find easily that the same statement holds for the case when $\left(x_{2}(t)-x_{1}(t)\right)<0$ and $\left(y_{2}(t)-y_{1}(t)\right)>0$.

Third we consider the solution $(x(t), y(t))$ in a neighborhood of $t=t_{0}$ such that $x_{2}\left(t_{0}\right)-x_{1}\left(t_{0}\right)=0$ and $\left(y_{2}\left(t_{0}\right)-y_{1}\left(t_{0}\right)\right)>0$. Consider the Dini derivatives of $\rho(t)$ at $t=t_{0}$. Then we have

$$
\begin{aligned}
\left.D^{-} \rho\right|_{t=t_{0}}= & -\left(y_{2}\left(t_{0}\right)-y_{1}\left(t_{0}\right)\right) \\
& -\left.\frac{1}{\rho\left(t_{0}\right)} \int_{x_{1}(t)}^{x_{2}(t)}\left(f(x)-\frac{d g}{d x}(t)\right) d x\right|_{t=t_{0}}<0 .
\end{aligned}
$$

On the other hand,

$$
\begin{aligned}
\left.\rho(t) D^{+} \rho\right|_{t=t_{0}}= & \left.\left(y_{2}\left(t_{0}\right)-y_{1}\left(t_{0}\right)\right) \int_{x_{1}(t)}^{x_{2}(t)}\left(1-\frac{d g}{d x}(x)\right) d x\right|_{t=t_{0}} \\
& -\left.\left(x_{2}\left(t_{0}\right)-x_{1}\left(t_{0}\right)\right) \int_{x_{1}(t)}^{x_{2}(t)} f(x) d x\right|_{t=t_{0}}=0 .
\end{aligned}
$$

So, we have Dini derivative of $D^{+} \rho(t)$.

$$
\left.D^{+} D^{+} \rho(t)\right|_{t=t_{0}}=\frac{I_{1}(t)}{\rho\left(t_{0}\right)}-\left.\frac{I_{2}^{2}(t)}{\rho^{2}\left(t_{0}\right)}\right|_{t=t_{0}} .
$$

Here

$$
I_{1}(t)=\frac{d I_{2}}{d t}(t)
$$

and

$$
\begin{aligned}
I_{2}(t)= & {\left[\left(x_{2}(t)-g\left(x_{2}(t)\right)\right)-\left(x_{1}(t)-g\left(x_{1}(t)\right)\right)\right] \times\left(y_{2}(t)-y_{1}(t)\right) } \\
& -\left(x_{2}(t)-x_{1}(t)\right)\left[F\left(x_{2}(t)\right)-F\left(x_{1}(t)\right)\right] .
\end{aligned}
$$

So, we must show $I_{1}(t)<0$. We can find easily,

$$
\left.I_{1}(t)\right|_{t=t_{0}}=\left.\left(y_{2}\left(t_{0}\right)-y_{1}\left(t_{0}\right)\right)\left[1-g^{\prime}\left(x_{1}(t)\right)\right]\right|_{t=t_{0}}<0 .
$$


Hence we find that the Dini derivative is never positive. The case $\left(y_{2}(t)-y_{1}(t)\right)$ $<0$ is shown by the same way.

Lastly we consider the solution $(x(t), y(t))$ in a neighborhood of $t=t_{0}$ such that $\left(y_{2}\left(t_{0}\right)-y_{1}\left(t_{0}\right)\right)=0$ and $\left(x_{2}\left(t_{0}\right)-x_{1}\left(t_{0}\right)\right)>0$. We have Dini derivative

$$
\begin{gathered}
\left.\rho(t) D^{-} \rho(t)\right|_{t=t_{0}}=\left.\left(y_{2}\left(t_{0}\right)-y_{1}\left(t_{0}\right)\right) \int_{x_{1}(t)}^{x_{2}(t)}\left(1-\frac{d g}{d x}(x)\right) d x\right|_{t=t_{0}} \\
-\left.\left(x_{2}\left(t_{0}\right)-x_{1}\left(t_{0}\right)\right) \int_{x_{1}(t)}^{x_{2}(t)} f(x)\right|_{t=t_{0}}<0 \\
\left.D^{+} \rho(t)\right|_{t=t_{0}}=\left(y_{2}\left(t_{0}\right)-y_{1}\left(t_{0}\right)\right)-\left.\int_{x_{1}(t)}^{x_{2}(t)}\left(f(x)-\frac{d g}{d x}(x)\right) d x\right|_{t=t_{0}}<0 .
\end{gathered}
$$

These show $\rho^{\prime}(t)$ is a decreasing function.

We finish the proof of Lemma 9.

\section{§4. Application.}

In this section, we show two corollaries. To this end, we need the following theorem by $K$. Shiraiwa :

Theorem (Shiraiwa) In the equation

$$
\begin{aligned}
& x^{\prime}=y \\
& y^{\prime}=-f(x) y-g(x)+e(t) .
\end{aligned}
$$

the following properties are assumed to be satisfied.

(a) There exists a solution of the equation (4.1) under any initial condition.

(b) There exist positive constants $C$ and $E$ such that

$$
f(x) \geqq C, \quad|e(t)| \leqq E .
$$

(c) $g(x)$ is a differentiable function satisfying the following conditions (i), (ii) and (iii):

(i ) $\quad d g(x) / d x$ is bounded on any finite interval.;

(ii) $\quad d g(x) / d x \geqq 0$;

(iii) $\quad \lim _{x \rightarrow \infty} g(x)>E, \quad \lim _{x \rightarrow-\infty} g(x)<-E$.

By the condition $(c), g(x)$ is a monotone increasing function, and there exist numbers $x_{1}$ and $x_{2}\left(x_{1}<x_{2}\right)$ such that

$$
g\left(x_{1}\right)=-E, \quad g\left(x_{2}\right)=E .
$$

Let $x(t)$ be any solution of the equation (4.1). Then there exists a large $t_{0}$ such that 


$$
x_{1}-\frac{4 E}{C} \leqq x(t) \leqq x_{2}+\frac{4 E}{C}
$$

and

$$
\left|x^{\prime}(t)\right| \leqq \frac{4 E}{C}
$$

for $t>t_{0}$.

In this paper, there is no need that the condition (a) is satisfied, because of the assumption (1.3). This gives the following corollary to Theorem 8.

COROLLARY 10. Suppose that the equation (1.1) satisfies conditions (b) and (c), and suppose that we have $\alpha$ and $\beta$ such that

$$
g(\alpha)=-E, \quad g(\beta)=E
$$

hold for the equation (1.1). Then the equation (1.1) has only one completely stable periodic solution with the least period $L$.

Moreover, making stronger the condition of Theorem 8 , we have the following corollary.

COROLLARY 11. We assume that the function $f(x)$ and $g(x)$ in the equation (1.1) satisfy

$$
\begin{array}{ll}
f(x)>C & \text { for }{ }^{\forall} x \in R \\
\frac{d g}{d x}(x) \geqq 0 & \text { for }{ }^{\forall}|x|>X \\
x g(x)>0 &
\end{array}
$$

for sufficiently large $X>0$. And

$$
E<\left|g\left(x_{\imath}\right)\right|<3 E
$$

where $x_{\imath}$ is the point that satisfy $d g\left(x_{\imath}\right) / d x=0$. Let $\alpha_{\imath}, \beta_{i}$ be that

and

$$
\begin{aligned}
& g\left(\alpha_{\imath}\right)=-E \\
& g\left(\beta_{\imath}\right)=E
\end{aligned}
$$

$$
\alpha_{1}<\beta_{1}<\beta_{2}<\alpha_{2}<\cdots<\alpha_{2 j}<\alpha_{2 j+1}<\beta_{2 j+1}<\beta_{2 j+2}<\alpha_{2 j+2}<\cdots<\alpha_{2 m+1}<\beta_{2 m+1} .
$$

Let us assume that $|g(x)| \neq E$ for $x \neq \alpha_{\jmath}, \beta_{\jmath}$. Moreover, we assume that the assumption of Theorem 8 holds in the region $\alpha_{2 j+1}-4 E / C^{2}<x<\beta_{2 j+1}+4 E / C^{2}$. Then the equation (1.1) has just one completely stable periodic solutions in each region

$$
D s_{j}=\left\{(x, y)\left|\alpha_{2 j+1}-\frac{4 E}{C^{2}}<x<\beta_{2 j+1}+\frac{4 E}{C^{2}},\right| y \mid<\frac{4 E}{C}\right\},
$$


and the equation (1.1) has just one directly unstable periodic solutions in each region

$$
D u_{\jmath}=\left\{(x, y)\left|\beta_{2 j+2}<x<\alpha_{2 j+2},\right| y \mid<\frac{4 E}{C}\right\} .
$$

And, there exist just $m+1 D s$, and just $m$ Du. Then there exist just $m+1$ periodic solutions which are completely stable and just $m$ periodic solutions which are directely unstable.

Proof of Corrolary 11. First, we prove that there exist no periodic solution outside of region

$$
D=\left\{(x, y)\left|\alpha_{1}-\frac{4 E}{C^{2}}<x<\beta_{2 m+1}+\frac{4 E}{C^{2}},\right| y \mid<\frac{4 E}{C}\right\}
$$

where $y=x^{\prime}$.

$C_{1}$ is the solution of the equation (2.6) started from $\left(\beta_{2 m+1}, r\right)$ to a intersection with $x$-axis at $\left(X_{1}, 0\right)$. Here, $r$ is larger than $4 E / C$. Next we define $C_{2}$ as the solution of the equation $(2.7)$ from $\left(X_{1}, 0\right)$ to the point where it meets with the curve $-C y-g(x)-E=0$. We call it $\left(X_{2}, Y_{2}\right)$. Here, we suppose the lemma.

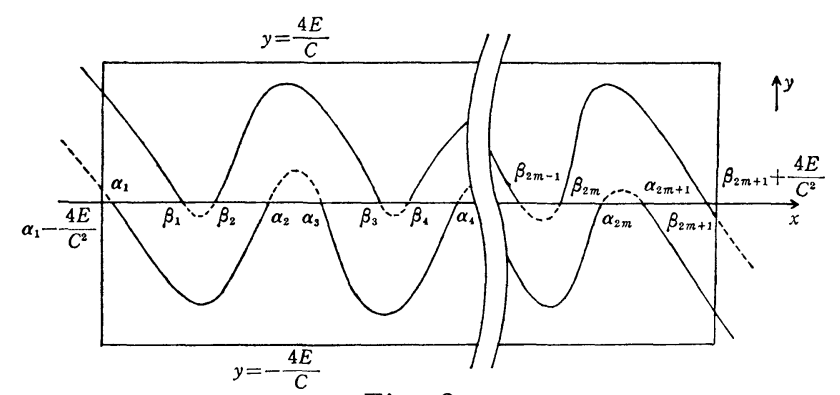

Fig. 2.

LEMMA 12. The point $\left(X_{2}, Y_{2}\right)$ satisfies $Y_{2} \geqq-r$.

From this lemma, we have $Y_{2} \geqq-r$, then we define $C_{3}$ as the line segment from $\left(X_{2}, Y_{2}\right)$ to $\left(X_{2},-r\right)$. Next we make the curve $C_{4}$, which is the solution of the equation (2.7) starting from $\left(X_{2},-r\right)$ till $x=\alpha_{1}$. The end point is $\left(\alpha_{1}, Y_{3}\right)$. It is clear that

$$
\min _{\beta_{2 m+1<x} \leqq X_{2}} \frac{-g(x)-E}{C} \geqq-r
$$

and

$$
\min _{x<\beta_{2 m+1}} \frac{-g(x)-E}{C}>-\frac{4 E}{C} .
$$

So the solution of the equation (2.7) is decreasing for $x \leqq X_{2}$. Then we 
have $Y_{3}>-r$. At the end, we define $C_{5}$ which is a line segment from $\left(\alpha_{1}, Y_{3}\right)$ to $\left(\alpha_{1},-r\right)$. And we assume the following lemma for drawing the curve $C_{6}-C_{10}$ by the same way.

LEMMA 13. The point $\left(X_{5}, Y_{5}\right)$ satisfies $Y_{5} \leqq r$.

We consider the tangent vectors of the solutions of the equation (2.4) on these curves $C_{1}, C_{2}, C_{4}, C_{6}, C_{7}$ and $C_{9}$. From the equations (2.8) and (2.9), we find that they turn to inside of this closed curve. For $C_{3}, C_{5}, C_{8}$ and $C_{10}$, the first equation (2.3) shows that the tangent vector direct inside of it. Hence all solutions of the equation (2.4) enter the domain $D$.

Next, we define subregion as follows:

$$
\begin{aligned}
& D 1_{\jmath}=\left\{(x, y)\left|\alpha_{2 j+1}-\frac{4 E}{C^{2}}<x<\beta_{2 j+1}+\frac{4 E}{C^{2}},\right| y \mid<\frac{4 E}{C}\right\} \\
& D 2_{\jmath}=\left\{(x, y) \mid \beta_{2 j+1}+\frac{4 E}{C^{2}} \leqq x \leqq \beta_{2 j+2}, 0 \leqq y<\frac{4 E}{C}\right\} \\
& D 3_{\jmath}=\left\{(x, y) \mid \beta_{2 j+1}+\frac{4 E}{C^{2}} \leqq x \leqq \beta_{2 j+2},-\frac{4 E}{C}<y \leqq 0\right\} \\
& D 4_{j}=\left\{(x, y)\left|\beta_{2 j+2}<x<\alpha_{2 j+2},\right| y \mid<\frac{4 E}{C}\right\} \\
& D 5_{\jmath}=\left\{(x, y) \mid \alpha_{2 j+2} \leqq x \leqq \alpha_{2 j+3}-\frac{4 E}{C^{2}}, 0 \leqq y<\frac{4 E}{C}\right\} \\
& D 6_{\jmath}=\left\{(x, y) \mid \alpha_{2 j+2} \leqq x \leqq \alpha_{2 j+3}-\frac{4 E}{C^{2}},-\frac{4 E}{C}<y \leqq 0\right\} .
\end{aligned}
$$

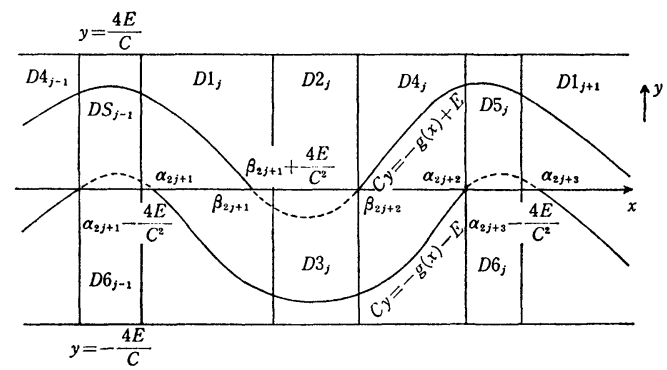

Fig. 3.

We consider the tangent vector of any solution of the equation (2.4) on $|y|=$ $4 E / C$. We find that the solution is decreasing. And any solution of the equation (2.4) moves from left to right above $x$-axis and inversely below $x$-axis. Hence, the behaivor of solution of the equation (2.4) on $x$-axis shows the existence of periodic solution. But in the domain $D 1_{\jmath}$, there exists only one completely stable periodic solution by Theorem 8 . On $\beta_{2 j+1}+4 E / C^{2} \leqq x \leqq \alpha_{2 j+2}, y$ 
component of the tangent vector is negative. So, any solution in the domain $D 2$, goes to the domain $D 3$, or $D 4$. And any solution in the domain $D 3$, goes to $D 1_{j}$. By the same way, we find that any solution in $D 5_{j}$ goes to $D 1_{j+1}$, and any solution in $D 6$, goes to $D 4_{j}$ or $D 5_{j}$. Next we show that there is only one periodic solution in the domain $D 4$.

Let $\bar{x}(t)$ be a periodic solution of the equation (1.1) in $\beta_{2 j+1} \leqq x \leqq \beta_{2 j+2}$, and let $x(t)$ be a solution of the equation (1.1) near $\bar{x}(t)$. We consider the system

$$
\begin{aligned}
& x^{\prime}=y-F(x) \\
& y^{\prime}=-g(x)+e(t) .
\end{aligned}
$$

Let $(\bar{x}(t), \bar{y}(t))$ and $(x(t), y(t))$ be the solution of the equation (2.4) corresponding to $\bar{x}(t)$ and $x(t)$ respectively. Let us calculate a derivative of $x(t)-\bar{x}(t)$ and $y(t)-\bar{y}(t)$. Then we have

$$
\begin{aligned}
& x^{\prime}(t)-\bar{x}^{\prime}(t)=\left(y(t)-\bar{y}^{\prime}(t)\right)-\int_{\bar{x}(t)}^{x(t)} f(u) d u \\
& y^{\prime}(t)-\bar{y}^{\prime}(t)=-\int_{\bar{x}(t)}^{x(t)} g^{\prime}(u) d u .
\end{aligned}
$$

We denote the tangent vector of $(x(t)-\bar{x}(t), y(t)-\bar{y}(t))$ by $(u, v)$. The above equation show that $u$ is positive above the curve:

$$
y(t)-\bar{y}(t)=\int_{\bar{x}(t)}^{x(t)} f(u) d u,
$$

and $u$ is negative below the curve. And we find that $v$ is positive in the left of the line $x(t)=\bar{x}(t)$, and negative in the right of the line $x(t)=\bar{x}(t)$. So, we have that $(x(t), y(t))$ go out from the region $\beta_{2 j+1} \leqq x \leqq \beta_{2 j+2}$. Hence, we find at most one periodic solution in the region $\beta_{2 j+1} \leqq x \leqq \beta_{2 j+2}$.

Then there exist at least $2 m+1$ periodic solutions, and there exist just $m+1$ periodic solutions whitch are completely stable. Moreover, Index Theorem of Levinson [3] shows that if there exist $m+1$ completely stable periodic solutions then there exist at least $m$ directly unstable periodic solutions. So we find just $m$ periodic solutions that are directly unstable. We finish the proof of Corrolary 11.

Proof of Lemma 12. This proof is done by the same way as the proof of Lemma 6. When $x_{2} \leqq \beta_{2 j+1}$, it is clear that $Y_{2}>-4 E / C$ from $g(x)<E$, because $\left(X_{2}, Y_{2}\right)$ is the cross point of $-C y-g(x)-E=0$ and the solution of the equation (2.7). So we must show the lemma for $X_{2}>\beta_{2 j+1}$. From Lemma 3, we know that $Y_{2}$ is the minimum of $y$ of $C_{2}$.

Now we integrate the equation (2.6) from $\left(\beta_{2 j+1}, r\right)$ to $\left(X_{1}, 0\right)$. So we have

$$
-\frac{r^{2}}{2}=-C \int_{0}^{X_{1}} y d x-G\left(X_{1}\right)+G\left(\beta_{2 j+1}\right)+E\left(X_{1}+\beta_{2 j+1}\right),
$$


where

$$
G(x)=\int_{0}^{x} g(u) d u .
$$

$C_{1}$ is a solution of the equation:

$$
y^{2} \frac{d^{2} y}{d x^{2}}=(g(x)-E) \frac{d y}{d x}-\frac{d g}{d x}(x) y,
$$

so it is convex upward. Then the integral part of the equation (4.4) is greater than the area of triangle whose vertices are $\left(\beta_{2 j+1}, \gamma\right),\left(\beta_{2 j+1}, 0\right)$ and $\left(X_{1}, 0\right)$. Hence, we find

$$
-\frac{r^{2}}{2} \leqq-C \frac{r}{2}\left(X_{1}-\beta_{2 j+1}\right)-G\left(X_{1}\right)+G\left(\beta_{2 j+1}\right)+E\left(X_{1}-\beta_{2 j+1}\right) .
$$

On the other hand, we integrate the equation (2.7) from $\left(X_{1}, 0\right)$ to $\left(X_{2}, Y_{2}\right)$. Then we have

$$
\frac{Y_{2}^{2}}{2}=-C \int_{X_{1}}^{X_{2}} y d x-G\left(X_{2}\right)+G\left(X_{1}\right)-E\left(X_{2}-X_{1}\right) .
$$

The integrated part of the equation (4.8) is positive when $y<0$ and $X_{2}<X_{1}$. Therefore the equation (4.8) gives

$$
\frac{Y_{2}^{2}}{2} \leqq-G\left(X_{2}\right)+G\left(X_{1}\right)-E\left(X_{2}-X_{1}\right) .
$$

We add the equation (4.7) to the equation (4.9). So we find

$$
\begin{aligned}
\frac{\left(Y_{2}^{2}-r\right)}{2} & \leqq-C \frac{r}{2}\left(X_{1}-\beta_{2 j+1}\right)-G\left(X_{2}\right)+G\left(\beta_{2 j+1}\right)+E\left[\left(X_{1}-\beta_{2 j+1}\right)+\left(X_{1}-X_{2}\right)\right] \\
& \leqq\left(-C \frac{r}{2}+2 E\right)\left(X_{1}-\beta_{2 j+1}\right)-\int_{\beta_{2 j+1}}^{X_{2}} g(u) d u \leqq 0 .
\end{aligned}
$$

We have

$$
\int_{\beta_{2 \jmath+1}}^{x_{2}} g(u) d u \leqq 0
$$

from the condition

$$
\begin{aligned}
& g\left(\beta_{2 j+1}\right)=E \\
& \frac{d g}{d x}(x) \geqq 0 \quad \text { for } x \geqq \beta_{2 j+1},
\end{aligned}
$$

and $\left(X_{1}-\beta_{2 j+1}\right)>\left(X_{1}-X_{2}\right)$. Hence, we find

$$
Y_{2}^{2}-r^{2} \leqq 0
$$

After all, we have $\left|Y^{2}\right| \leqq r$, so the proof of Lemma 12 is completed. Lemma 13 is proved as the same way as the proof of Lemma 12 . 


\section{§5. Examples.}

In this section, we give two examples and their numerical results are compared with the theory.

First, we consider

From this

$$
\begin{aligned}
& f(x)=0.1 x^{2}+1 \\
& g(x)=x^{3}-x \\
& e(t)=0.1 \cos 2 \pi t
\end{aligned}
$$

$$
\frac{d g}{d x}(x)=3 x^{2}-1
$$

So, we have $d g / d x(x)>1$ in:

$$
x>\sqrt{\frac{2}{3}} \quad x<-\sqrt{\frac{2}{3}}
$$

and $f(x) \geqq d g / d x(x)$ is satisfied in :

$$
-\sqrt{\frac{20}{29}} \leqq x \leqq \sqrt{\frac{20}{29}}
$$

Thus, we have two completely stable periodic solutions in the following regions:

$$
-\sqrt{\frac{20}{29}} \leqq x<-\sqrt{\frac{2}{3}} \quad \sqrt{\frac{2}{3}}<x \leqq \sqrt{\frac{20}{29}}
$$

respectively. The numerical results are indicated in Fig. 4, 5, 6. We can see

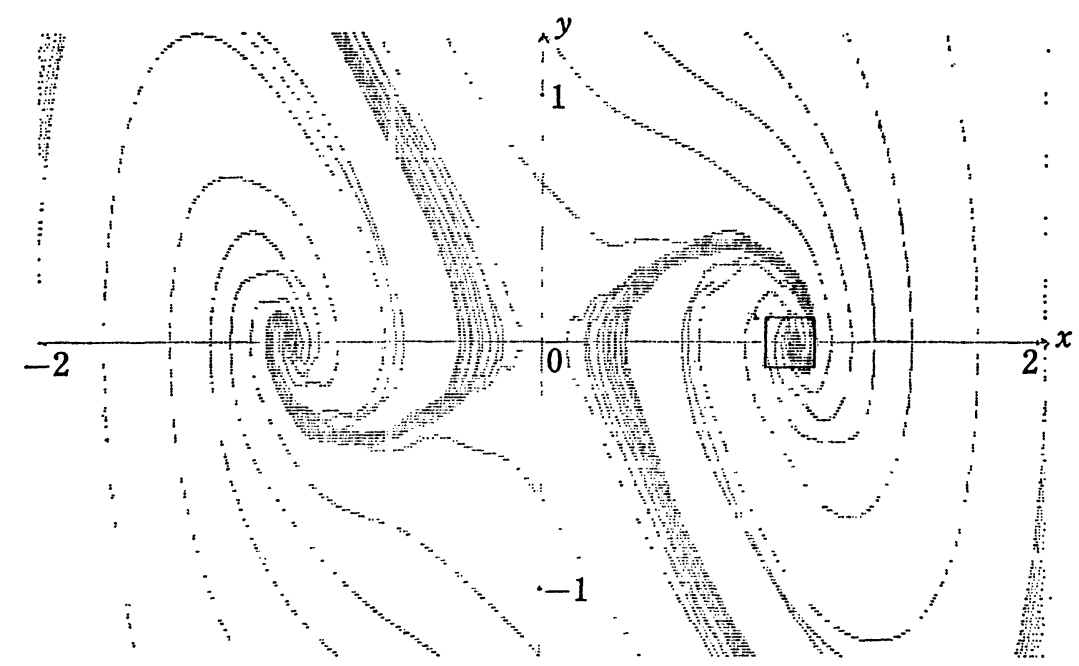

Fig. 4. 
two completely stable periodic solutions. We have a directly unstable periodic solution near origin by theory, but we can not find by calculation.

Next example is

$$
\begin{aligned}
& f(x)=10 x^{2}+1.7 \\
& g(x)=0.02 x^{5}-0.33 x^{3}+0.9 x \\
& e(t)=0.5 \cos 2 \pi t .
\end{aligned}
$$

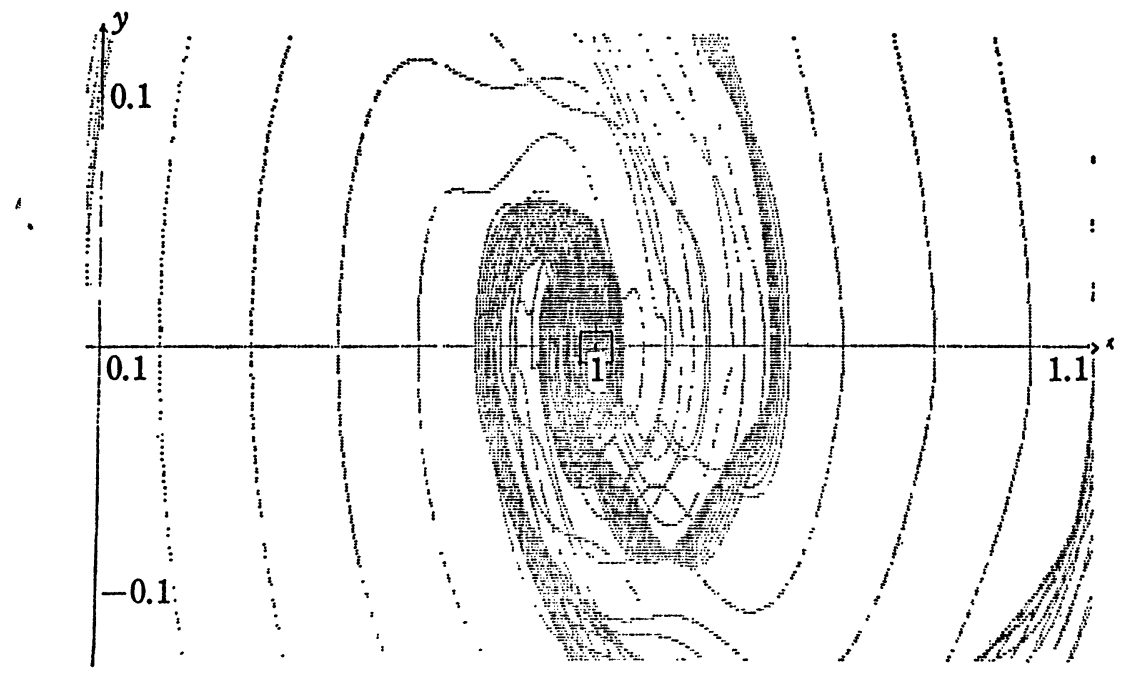

Fig. 5.

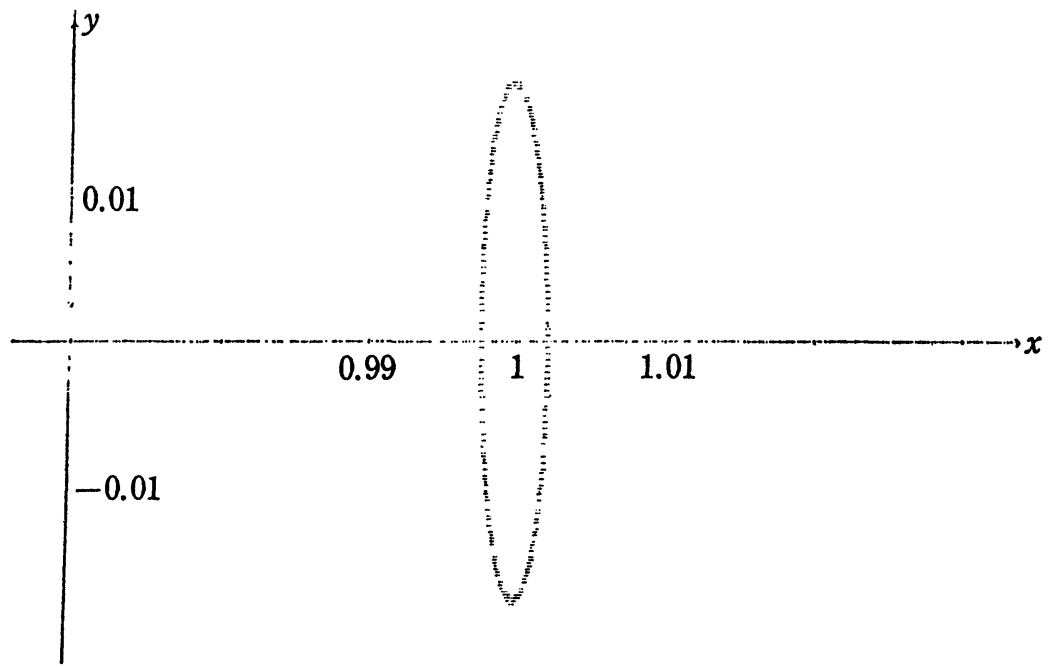

Fig. 6. 
In this example, we can find three completely stable solutions near $x=-3.5$, 0, 3.5. And, we can confirm three completely solutions. See Fig. 7.

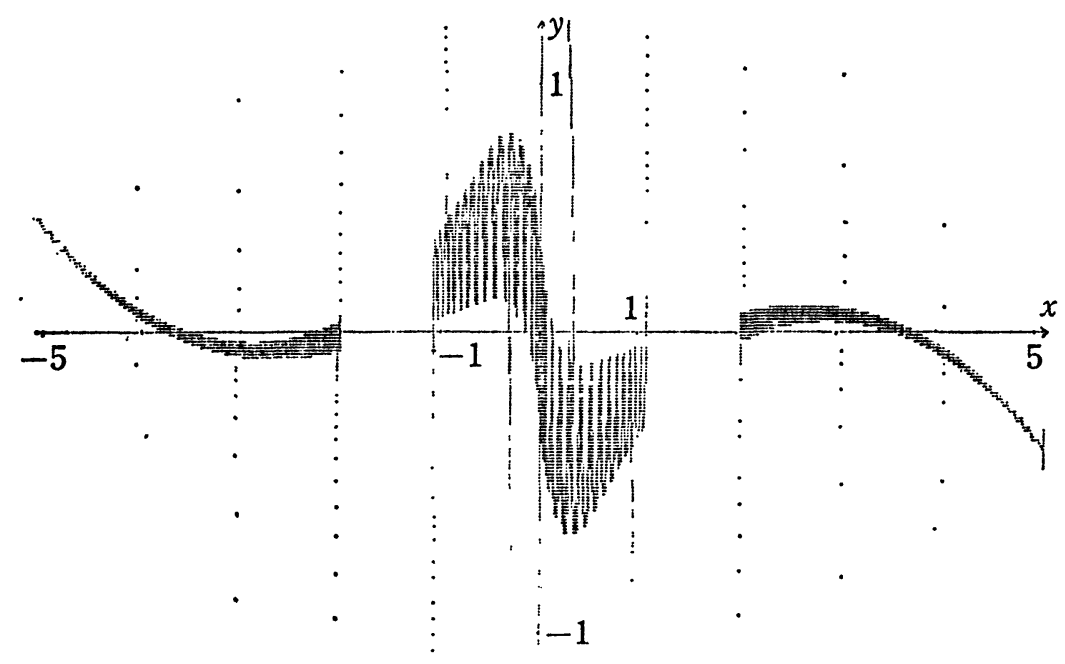

Fig. 7.

\section{REFERENCES}

[1] N. Levinson, On the Existence of Periodic Solutions for Second Order Differential Equations with the Forcing Term, J. Math. Phys., vol. 22 (1943), pp. 41-48.

[2] N. Levinson, On a Non-Linear Differential Equation of Second Order, J. Math. Phys., vol. 22 (1943), pp. 41-48.

[3] N. Levinson, Transformation Theory of Non-Linear Differential Equations of the Second Order, Ann. of Math., vol. 45, No. 4 (1944), pp. 723-737.

[4] W.S. Loud, Boundedness and Convergence of Solutions of $x^{\prime \prime}+c x^{\prime}+g(x)=e(t)$, Duke Math. J., vol. 24 (1957), pp. 63-72.

[5] K. Shiraiwa, Boundedness and Convergence of Solutions of Duffing's Equation, Nagoya Math. J., vol. 66 (1977), pp. 151-166.

Department of Mathematics

TOKyo Institute of TECHNOLOGY 